\title{
1 Evaluating quality of implementation in physical activity interventions \\ 2 based on theories of motivation: Current challenges and future \\ 3 directions
}

4

5

6

The evidence base pointing towards the maladaptive health consequences of an inactive lifestyle highlights the need for interventions that are effective in changing and maintaining physical activity behaviours. Theories of motivation are frequently applied to inform the content and delivery of such interventions. Systematic monitoring and evaluation of the quality of intervention implementation is therefore an important step in understanding if and how theories of motivation can be adopted and effectively applied to promote and/or sustain physical activity behaviours. However, intervention implementation quality in studies that aim to apply motivation theory to promote physical activity is often under-reported. The purpose of this article is firstly to review contemporary approaches used to monitor and evaluate intervention implementation. We outline the degree to which these methods have been used effectively in research concerned with applying theories of motivation to impact physical activity behaviours. Secondly, we identify and discuss specific challenges in effectively measuring quality of implementation faced by researchers that adopt a motivation theory basis to their work. Finally, recommendations for methods to monitor and evaluate intervention implementation in future trials aiming to promote physical activity based on theories of motivation are also proposed.

Keywords: Fidelity; process evaluation; implementation; intervention; physical activity

There is strong evidence that physical inactivity is one of the leading causes of ill-health and premature death in Western societies (Kohl et al., 2012). However, despite these extreme health risks, physical inactivity remains a global health problem. Thus, identifying the most effective means to promote and sustain regular physical activity is 
1 imperative for national governments and public health organisations to prevent chronic

2 illness and promote good health. As a consequence, there has been a significant increase

3 in studies that have developed and tested behavioural interventions designed to promote

4 physical activity. Such work is often grounded in theories of motivation (Biddle,

5 Mutrie, \& Gorely, 2015; Rhodes \& Dickau, 2012). Motivation has been identified as a

6 key construct determining the intensity and direction of action in human behaviour.

7 High quality motivation is purported to initiate, regulate, and sustain health behaviours

8 (Deci \& Ryan, 2000). Theories of motivation highlight the social-psychological

9 antecedents of optimal and sustainable motivation for a targeted behaviour, such as

10 physical activity. The inclusion of motivation theory in the development and testing of

11 interventions aiming to change or maintain physical activity behaviours is, therefore,

12 important (Conner \& Norman, 2015; Michie et al., 2008). In this review we aim to

13 evaluate contemporary approaches to the study of intervention implementation. We will

14 discuss challenges and possible solutions associated with assessment and reporting of

15 fidelity and quality of intervention implementation. We focus on studies that have

16 adopted and applied theories and models of motivation derived from social psychology

17 to inform intervention content. However, general issues relating to implementation

18 quality are relevant to any type of intervention, irrespective of whether they are guided

19 by motivational theory or not.

20 The development and testing of theory- and evidence-based behavioural

21 interventions is an important step in translating evidence from intervention research to

22 'real-world' practice. The study of implementation efficacy in controlled settings may

23 be useful for establishing the potential of an intervention to be effective. However,

24 testing effectiveness in 'real-world' conditions potentially has more value in informing

25 translation; an effective intervention must be able to operate within the contextual 
1 constraints that would characterise the 'real-world' setting (Michie, 2008). Fidelity is

2 more likely to be challenged in real-life settings due to the likely uncontrollability of

3 external factors. It is, therefore, potentially even more critical that implementation is

4 studied in these cases as the conclusions drawn from such interventions will only be

5 valuable if the degree to which content and quality of delivery implemented during

6 testing remains true to the intended design.

$7 \quad$ Nearly twenty years ago, Dane and Schneider (1998) reported that interventions

8 that deviated most from the original design protocol were the least effective.

9 Unfortunately the omission of assessment of implementation fidelity, or in other words,

10 whether intervention delivery is consistent with the intended design (Dusenbury,

11 Brannigan, Falco, \& Hansen, 2003), is an on-going major methodological limitation in

12 health promotion intervention research in the physical domain (Bellg et al., 2004;

13 Marcus et al., 2006; Naylor et al., 2015).

Broadly speaking, physical activity intervention research based on theories of

motivation aims to increase motivation in individuals to initiate and sustain health-

16 related physical activity behaviour. This may be achieved using specific techniques and

17 strategies linked to variables found to correlate with behaviour in formative research to motivate individuals to change their behaviour. There are a number of types of theories of motivation that have been used to understand physical activity behaviour and used as the basis for interventions. For example, interventions based on social cognitive theories

21 such as social cognitive theory (Bandura, 1986) and the theory of planned behaviour

22 (TPB; Ajzen, 1991), typically utilise persuasive techniques to manipulate individuals 23 beliefs and attitudes with respect to the target behaviour. In the case of physical activity 24 interventions, the TPB has been applied in clinical settings (Latimer, Ginis, \& Arbour, 25 2006) as well as non-clinical contexts such as schools (Chatzisarantis \& Hagger, 2005), 
1 and the workplace (Bardus, Blake, Lloyd, \& Suzanne Suggs, 2014). Another theory

2 derived from humanistic approaches to motivation, self-determination theory (SDT;

3 Deci \& Ryan, 1985, 2000), has tended to focus on influencing the style content and

4 style of communication of social agents and significant others to facilitate physical

5 activity participation, such as healthcare staff (e.g., Murray et al., 2015), exercise

6 instructors (Ntoumanis, Thøgersen-Ntoumani, Quested, Hancox, 2016) and sport

7 coaches (e.g., Duda et al., 2013). The aforementioned studies illustrate ways in which

8 motivation theories have been adopted in intervention research to promote motivation

9 toward physical activity behaviour. The advantage of adopting a theoretical basis to an

10 intervention over an atheoretical or a theory 'inspired' approach is that it provides a

11 framework for falsification and to systematically evaluate the processes and

12 mechanisms responsible for change (Michie \& Abraham, 2004). This enables

13 intervention researchers to identify the key components of interventions that are

14 effective in promoting motivation relative to those that lack effectiveness.

Although a theoretical underpinning is frequently advocated as essential in

16 health behaviour intervention design (Craig et al., 2008), a significant number of

17 physical activity interventions are atheoretical, or vary in the extent or way in which

18 theory has been applied in design and effectiveness evaluation (Prestwich et al., 2014).

19 Moreover, in studies that claim to be based on theory, the evidence supporting the

20 assumed association with effectiveness is unclear (Prestwich et al., 2014). The specific

21 ways that theory is utilised in intervention design and effectiveness testing may

22 determine the degree to which one or more components from theory can improve the

23 effectiveness of an intervention relative to the absence of those components. Without

24 sufficient study of implementation processes it is difficult to identify exactly how

25 theory-based components adopted in physical activity interventions are effective in 
1 promoting motivation to promote engagement in physical activity behaviour. Moreover,

2 the seldom reporting of how interventions are developed or the rationale that informed

3 key decisions made in this process (Hoddinott, 2015) further inhibits any potential for

4 identifying implementation-related moderators of intervention effectiveness.

The purpose of this paper is not to provide a review of intervention studies that

6 have attempted to promote physical activity via applying theories and models of

7 motivation. Rather, our goal is to highlight the importance of the assessment and

8 reporting of fidelity and quality of intervention implementation in these studies with

9 respect to their theoretical content. First, we outline the value of assessing intervention

10 implementation and its component parts in theory-based intervention research in

11 physical activity contexts. An overview of how implementation and associated terms

12 are currently operationalised in the wider health promotion literature (beyond physical

13 activity promotion), and the inconsistencies in this application, are discussed. Second,

14 we highlight the challenges associated in studying intervention implementation in

15 research drawing from theories and models of motivation. Finally, we offer some future

16 research directions and recommendations. While we focus on physical activity

17 interventions, we believe that the points raised have relevance to intervention research

18 on health behaviours more broadly.

19 Intervention Implementation in Physical Activity Promotion Research

20 In a systematic review of 30 intervention studies designed to promote physical

21 activity or dietary change, Greaves et al. (2011) reported none to assess the fidelity of

22 the intervention. This finding highlights that fidelity is not considered a priority by

23 authors and reviewers and its omission does not preclude publication of intervention

24 results. The void in examining intervention implementation is not unique to studies 
1 designed to promote physical activity and has been noted as a limitation in the wider

2 health promotion literature (Marcus et al., 2006).

As a further illustration, we conducted a literature search of electronic databases

4 (Scopus, Web of Science) in order to get a broad overview of the extent to which

5 theory-based, motivation-focused physical activity intervention studies have reported in

6 detail on the assessment and monitoring of implementation of theory in the testing of an

7 intervention. We initially searched for articles that referred to ('physical activity' or

8 'sport' or 'exercise' or 'PE' or 'Physical Education') and 'intervention' and

9 'motivation' and 'theory' within the title, keywords or abstract. Our search returned 485

10 articles. To further narrow the search to those studies that were or had included a

11 detailed account of assessment and/or monitoring of implementation, we repeated the

12 search and also specified 'intervention implementation' or 'feasibility' or 'fidelity' in

13 addition to the aforementioned terms. This returned 24 articles. This illustration

14 indicates that less than $5 \%$ of published work concerning interventions in the physical

15 activity domain has provided a mention of intervention implementation and fidelity in

16 the title, keywords or abstract. Other studies may have included examination of

17 intervention implementation but not referred to it in the title, keyword or abstract.

18 However, this point supports the argument that intervention implementation was not

19 considered a significant focus in the majority of the identified studies.

20 We recognise that some studies that may not have come up in our search may

21 still include a concise section detailing the study of implementation (e.g., Cohen,

22 Morgan, Plotnikoff, Callister, \& Lubans, 2015; Smith et al., 2014). In circumstances in

23 which a detailed process evaluation is not feasible, this may be a reasonable

24 compromise that would enable the reader to interpret the findings with necessary and

25 relevant background information. However, our search highlights the limited number of 
1 studies that dedicate major focus to the study of intervention implementation, relative to

2 the overall number of trials utilising theories of motivation to inform physical activity

3 interventions. There are numerous reasons why this may be the case and later in the

4 paper we discuss a range of issues that may preclude researchers from evaluating, or

5 editors from publishing, detailed accounts of the evaluation of intervention

6 implementation.

7 Terminology and Method in Intervention Implementation Research

The study of intervention implementation involves continuous evaluation and

9 monitoring of an intervention to identify the content delivered, how it is delivered, and

10 the degree to which the content delivery is aligned with the intended design (Borrelli,

11 2011; Dusenbury et al., 2003). Intervention implementation could be considered as a

12 moderator of the effect of an intervention on outcomes. Hence, it is critical in

explaining findings of intervention-based research (Moore et al., 2015). Evaluating

intervention implementation also makes it possible to determine whether a null finding could be attributable to a poor quality intervention, or to poor or inconsistent quality in the delivery. The latter is known as 'type III' error (Basch \& Gold, 1985; Dusenbury et al., 2003). It is, therefore, important to evaluate intervention implementation to ensure that high quality interventions with the potential to be effective are not disregarded on account of poor delivery.

A number of evaluation frameworks provide intervention researchers with a

21 starting point around which to frame the study of implementation. These frameworks

22 include approaches designed to assess and monitor implementation in trials of a wide

23 range of public health interventions (e.g., RE-AIM; Glasgow, Vogt, \& Boles, 1999).

24 Frameworks have also been published that aim to better target certain types of

25 intervention, such as those specifically targeting behaviour change (e.g., Borrelli, 2011). 
1 Recently, Moore et al., $(2014,2015)$ have proposed means to address the problems of

2 studying implementation in complex interventions (i.e., interventions with multiple

3 interacting factors; Craig et al., 2008), which may be overlooked by other frameworks

4 that do not inherently address interactions between different factors within or across

5 levels of intervention. Each approach identifies specified 'components' that can be

6 evaluated to ascertain quality of intervention implementation; interestingly, some

7 common terms and themes can be identified across all three frameworks. Yet these

8 frameworks also show diversity in how terms and themes are operationalised in relation

9 to one another and in practice. Across these frameworks as well as the wider health

10 promotion literature, there is a notable lack of consensus in the definition and

11 operationalisation of terms related to the monitoring and evaluation of intervention

12 implementation. This has resulted in diversity of opinions in what are considered to be

13 the core components and the priorities in this process, as well as how it should be

14 undertaken and reported. Moreover, inconsistencies in quality and consistency of term

15 definitions precludes researchers from reliably comparing results or conducting meta-

16 analyses (Naylor et al., 2015).

17 The RE-AIM Framework

18 The impediment on the overall progress of health promotion research caused by

19 inconsistent language and methods in evaluating interventions was first raised by

20 Glasgow et al. (1999). The RE-AIM (Reach, Effectiveness, Adoption, Implementation,

21 and Maintenance) framework was originally published to in an attempt to address this

22 issue of consistency. 'Reach' refers to the extent to which the target population are

23 willing to engage in the intervention. 'Effectiveness' captures the degree to which the

24 intervention has impacted upon the intended outcomes. 'Adoption' is defined as the

25 proportion of the target population who are responsible for implementation and willing 
1 to deliver the intervention. The quality and consistency of intervention delivery are

2 captured within 'implementation'. Finally, 'maintenance' refers to the degree to which

3 the intervention is sustained over time, both at the individual or organisational levels.

The RE-AIM framework may be useful in evaluating some facets of

5 implementation in certain theory-based public health interventions. For example, the

6 TPB is often used to inform the content of health behaviour messages displayed in

7 public places, such as stairwells at transport hubs (e.g., Lewis \& Eves, 2012). RE-AIM

8 could be utilised to inform evaluation of implementation in such interventions, via the

9 recording of the number of passengers who pass through the station who read the

10 message (reach), how many passengers who previously did not take the stairs changed

11 their behaviour subsequent to reading the message (effectiveness), how many stations

12 within a particular vicinity are willing to display the signage (adoption), to what extent

13 the signage is adopted and displayed as intended by transport hubs (implementation),

14 and how long the passengers continue to engage with the new behaviour (maintenance).

15 While this application of RE-AIM would be informative as to the overall effectiveness

16 of the intervention, it would not tease out whether the theory-based message content

17 was effective in changing the passengers' attitudes, perceived behavioural control or

18 intentions, and whether it was one or more of these mediating mechanisms that led to

19 changes in stair climbing behaviour.

20 When proposing RE-AIM, Glasgow and colleagues focused more specifically

21 on implementation of an intervention over a minimum one-year period (Glasgow et al.,

22 1999). It is also noteworthy that RE-AIM does not solely focus on intervention

23 implementation. Originally the framework was intended as a model for intervention

24 reporting, and more recently has been utilised to improve translation of research into

25 practice once the effectiveness of the intervention has been supported. Since its 
1 inception, RE-AIM has been applied across multiple intervention studies in the health

2 promotion field with over 300 publications comprising applications or discussions

3 concerning this framework currently listed on the RE-AIM website (http://www.re-

4 aim.hnfe.vt.edu/publications/index.html; accessed 05.11.2015).

\section{Borelli's (2011) Framework}

Since RE-AIM was proposed, other models and approaches to intervention

7 implementation and evaluation have been developed. For example, Borrelli (2011)

8 presented recommendations for best practice in treatment fidelity in relation to five key

9 aspects. These are study design, provider training, treatment delivery (i.e., the extent to

10 which the provider consistently delivered the treatment components (and not others)

11 with the required skill level), treatment receipt (i.e. the degree to which the intervention

12 was received by the participant as intended), and treatment enactment (i.e., whether the

13 participant could enact the required cognitive and behavioural strategies and skills).

14 Borrelli (2011) provide detailed tables listing strategies and recommendations as well as

15 a checklist that can be used to assess fidelity of treatment. These include six

16 considerations for treatment design (e.g., provide information about treatment dose),

17 seven principles for training of providers (e.g., assessment and monitoring of provider

18 skill maintenance over time), nine considerations for delivery of treatment (e.g., use of

19 a treatment manual), five recommendations for receipt of treatment (e.g., multicultural

20 factors considered in the development and delivery of the intervention), and two

21 criteria for the enactment of treatment skills (e.g., a strategy will be used to assess the

22 performance of the intervention skills in settings in which the intervention might be 23 applied).

24 The Application of Borrelli's (2011) Framework: A Motivational Interviewing $25 \quad$ Example 
Borrelli's strategies could be adapted to assess intervention implementation in

2 physical activity behaviour change studies. For example, researchers interested in the

3 impact of a theory-based motivational interviewing (Miller \& Rollnick, 2002)

4 intervention on physical activity behaviour change in cardiac rehabilitation patients

5 could enhance treatment fidelity at all of the study stages identified by Borrelli. At the

6 design stage, pilot work incorporating scope for patient feedback could be used to

7 identify the specific ways this communication style can be employed to motivate

8 physical activity behaviours among cardiac patients. For example, how, when and

9 where it would be appropriate for these patients to increase their physical activity

10 behaviours would be influenced by their physical health status and so this may influence

11 the types of changes the motivational interviewing process is guiding the patient

12 towards. Those strategies that most appropriately exemplify the core components of

13 motivational interviewing (i.e., engaging, evoking, increasing confidence, readiness and

14 desire for change, and planning for action) and that should be evident in the intervention

15 could be defined (Hardcastle, Fortier, Blake, \& Hagger, 2016). The factors that may

16 limit or alter the application of these communication techniques in a particular context

17 (e.g., fear of another cardiac event, anxious spouse) could also be identified during

18 piloting. Strategies to overcome such factors could be developed and incorporated into

19 the intervention to improve acceptability and feasibility. Clear and specific scripting

20 with context-specific examples could be created.

21 To date, many intervention studies grounded in motivational interviewing and

22 other perspectives of motivation do not report how providers are trained and any

23 attempts to improve fidelity via the training provided. The methods proposed by

24 Borrelli (2011) to enhance fidelity of provider training would be applicable to training

25 deliverers of motivational interviewing, as well as other motivation interventions, by 
1 standardising training, increasing 'buy in' of providers, and preventing drift or decay in

2 skills via 'top up' training and on-going feedback and mentoring. Better reporting of the

3 provider training protocol in future studies would help to increase knowledge with

4 regard to how much and exactly what type of training, mentoring and feedback is most

5 efficacious. Borrelli makes some suggestions of generic strategies that could be

6 employed to improve fidelity of delivery in a motivational interviewing intervention,

7 such as provision of delivery manuals, on-going supervision to identify and correct

8 mistakes in delivery, and determining 'acceptable' levels of competency for a provider

9 to be considered sufficiently trained. Other strategies could be specifically customised

10 to motivational interviewing. These could include coding audio and/or video footage of

11 patient interactions to determine the frequency of use of certain strategies, such as using

12 open-ended questions and providing positive affirmations. Coding tools could be

13 developed that reflect the core components of motivational interviewing and context-

14 specific applications of these strategies (Hardcastle et al., 2016).

Finally, Borrelli (2011) provides recommendations for enhancing fidelity of

16 receipt of the intervention by the patient. The suggestions tend to work on the

17 assumption that the intervention involves 'upskilling' the patients to engage in specific

18 behaviours. In the case of cardiac rehabilitation patients, principles from motivational

19 interviewing could be used to strengthen the potential impact of the proposed strategies.

20 For example, while it is recommended that the intervention is based around

21 achievement-related objectives, within a motivational interviewing intervention

22 providers could be trained to ensure that the focus is on directing the patient towards

23 setting their own physical activity-related objectives, reflecting the 'patient-led'

24 philosophy of motivational interviewing. In addition, aligned with Borrelli's

25 recommendations, interventionists could ensure that educational materials are engaging 
1 and contextually and culturally appropriate, but the language and style in how the

2 materials are presented could reflect the principles of motivational interviewing. Thus,

3 instead of simply providing such information, motivational interviewing-focused

4 materials could, for example, incorporate a series of questions that engage patients in

5 continuing to evoke change-related cognitions and in turn, behaviours.

\section{$6 \quad$ Medical Research Council Guidelines}

The model by Borrelli (2011) centres on the issue of preserving fidelity across

8 all of the five central domains, suggesting that fidelity is central to the process of

9 effective implementation. Indeed, in the literature, the study of intervention fidelity is

10 sometimes considered synonymous to undertaking a process evaluation (e.g., Robbins,

11 Pfeiffer, Wesolek, \& Lo, 2014). However, recent guidelines put forward by the UK

12 Medical Research Council (Moore et al., 2015) refer to process evaluation as being a

13 specific investigation that "aims to understand the functioning of an intervention, by

14 examining implementation, mechanisms of impact, and contextual factors" (Moore et

15 al., 2014, p. 8). Thus, according to this perspective, fidelity is only one aspect of

16 implementation, which is only one component of a process evaluation. Implementation

17 is defined as, "the process through which interventions are delivered, and what is

18 delivered in practice" (Moore et al., 2014, p. 8). Examining fidelity is one important

19 aspect of implementation but in isolation will not reveal a full picture of the

20 implementation process. According to Moore et al. (2014), implementation also

21 comprises the process ('the structures, resources and mechanisms through which

22 delivery is achieved'), adaptations ('alterations made to an intervention in order to

23 achieve better contextual fit'), dose ('how much intervention is delivered'), and reach

24 ('the extent to which a target audience comes into contact with the intervention') of an

25 intervention. A high quality intervention would also demonstrate fidelity (i.e., 
1 remaining true to design) in relation to dose, adaptations and process, as well as

2 intervention delivery. In other words, one might expect the designers to have specific

3 targets in relation to these facets of implementation. As such, aspects of the Borrelli

4 (2011), framework could be considered to be embedded within the Moore et al. (2015)

5 model.

Moore et al. (2015) proposed that a comprehensive process evaluation should

7 also incorporate assessment of the mechanisms of impact (i.e., how participants respond

8 to the intervention, potential moderators and mediators), and the context in which the

9 intervention is delivered (i.e., how contextual factors interact with how the intervention

10 works), as well as the interplay between components of implementation, context and

11 mechanisms. Collectively, these factors mediate the association between the

12 intervention itself and its outcomes. Moore et al. (2015) have therefore facilitated the study of implementation of complex interventions as their approach also considers the potential impact of contextual and individual factors that may interact with implementation. We concur with Moore et al that without considering mechanisms of

16 impact and the context in which the intervention is being delivered, evaluation of

17 intervention implementation alone will not fully explain if and how an intervention

18 relates to measured outcomes in a trial. It is also worth noting that many physical

19 activity mediation analyses produce null findings (Lubans, Foster, \& Biddle, 2008;

20 Rhodes \& Pfaeffli, 2010). However, the reporting of null findings is still important as

21 such details can serve to inform the design and delivery of future intervention studies.

\section{Motivation-specific Challenges in Selecting an Appropriate Framework}


1 intervention implementation may be formed by undertaking a full process evaluation,

2 such an evaluation is not always practical and is likely to be highly resource intensive.

3 The diversity and complexity of intervention studies also demands that there is

4 variability in the goals and methods of a process evaluation to suit each study (Moore et

5 al., 2015). For example, in a study testing an intervention that targets the

6 communication style of an exercise instructor to promote adaptive motivation among

7 exercisers (e.g., Ntoumanis et al., 2016) it may be challenging to assess all features of

8 implementation identified by Moore et al. (2015) or to apply the approaches to assess

9 fidelity of treatment receipt and enactment, as defined by Borrelli (2011). This is

10 because although the 'treatment' is ultimately targeting the quality of the physical

11 activity-related motivation of the exerciser, the intervention itself is a communication

12 skills education programme directed towards the exercise instructor. The instructor may

13 be trained in specific skills to incorporate into his or her communication style, but at the

14 exerciser level the hypothesised changes are cognitive or affective not behavioural, as

15 the exerciser is already physically active. Changes in the instructor's communication

16 style may be subtle alterations to phrasing, body language, or class content and

17 structure. This would be anticipated to have an overall impact upon the motivational environment in the exercise class. As such, there may be a change in quality, but not quantity of instructor behaviours. Thus, it becomes challenging to assess fidelity of receipt and enactment, or to assess 'dose' at the exerciser level. In this case then, the researcher may need to be selective in adopting the features of a process evaluation that make sense in the context of the underlying theory, participants targeted, and practical circumstances.

For example, in a self-determination theory-based intervention in a physical 
1 of the degree to which what is delivered be consistent with the theory (i.e., exercisers

2 are motivated in a manner that supports their autonomy, competence and relatedness).

3 This could be evaluated via the use of observation scales to tap the need supportive

4 features of the environment, and/or changes in participants' perceptions of their

5 instructors' need supportive behaviours. Contextual factors that might impact the

6 intervention delivery and effectiveness could include the size of exercise class, as it

7 could affect the degree of individual interaction between each instructor and individual

8 exerciser. Drawing from self-determination theory, mechanisms of impact of the

9 intervention upon exercise behaviour would be expected to include the exerciser's

10 degree of basic need satisfaction (i.e., feelings of autonomy, competence and

11 relatedness in the exercise setting), and motivation regulations (i.e., reasons) for

12 exercise. Pre- and post- assessments of these mechanisms would aid interpretation of

13 intervention effects and the utility of the theory in explaining the outcomes.

14 If a full process evaluation is not possible or appropriate in the case of all

15 interventions, a middle ground needs to be identified to determine a 'minimum

16 acceptable' level of implementation evaluation. In the case of motivation-based

17 intervention studies in the physical domain, it would be advantageous to identify which

18 elements of implementation evaluation are most valuable in identifying effectiveness

19 and efficacy of an intervention. This would be a worthwhile avenue for future research

20 and may need to be a theory-specific endeavour. There have been recent calls for more

21 detailed description of interventions, to facilitate replication and enable other

22 researchers to build on existing findings. Checklists such as TIDierR (Hoffmann et al.,

23 2014) and WIDER (Albrecht, Archibald, Arseneau, \& Scott, 2013) should serve to

24 improve the quality of intervention reporting which in turn will facilitate efforts to

25 effectively evaluate intervention implementation. 
Alongside highlighting the potential of several models of implementation when

2 applied with different theories, this section has also highlighted the on-going variability

3 in use of terminology. Key terms such as fidelity and implementation are used inter-

4 changeably, or defined and related to one another in different ways across frameworks.

5 Consequently, the aforementioned problem of inconsistency in use of terminology and

6 methodology that led to the original development of RE-AIM has, in fact, been

7 amplified. This variability in use of terminology creates significant challenges in

8 identifying consistently effective and ineffective intervention features across a number

9 of studies that have applied the same theory for the same purpose (e.g., the promotion of

10 physical activity). From the perspective of physical activity promotion research, it may

11 not be viable to propose a common framework to assess implementation as each

12 framework offers something slightly different and so the appropriate choice will depend

13 on the research question. However, the adoption of a common language of

14 implementation would be an important first step in moving towards quality control and

15 synergy in undertaking and reporting physical activity-related intervention studies.

\section{Challenges of Monitoring and Evaluating Intervention Implementation}

Many factors may contribute to the lack of emphasis on publishing implementation data in motivation-informed intervention research in physical activity

19 settings. One important issue is that of intervention complexity. Traditionally,

20 interventions designed to change physical activity focused on the provision of

21 information, and neglected to consider whether the targeted individuals had the physical

22 and psychological capacity to enact the targeted behaviour (Michie, van Stralen, \&

23 West, 2011). A growing body of research suggests that information alone is not

24 sufficient to change behaviour (Hagger \& Luszczynska, 2014). There is growing

25 support for the notion that interventions must also ensure that the individual has the 
1 psychological capacity, social and contextual opportunity but also critically, the

2 motivation to initiate and sustain the targeted behaviour in the face of more attractive

3 alternatives (Biddle et al., 2015). Correspondingly, interventions that aim to change

4 physical activity behaviour are becoming increasingly complex. For example,

5 interventions based on motivation theory aim to impact physical activity behaviour at

6 the individual level (e.g., the newly signed up exercise participant) by changing the

7 motivational style of salient social agents in the individuals' environment with whom

8 they regularly interact (e.g., the exercise instructor). To be effective, the intervention

9 should change and maximise not only the quality of the instruction provided, but also

10 stimulate the social-psychological mechanisms known to initiate and regulate the

11 individuals' physical activity behaviour (e.g., beliefs, habits) (Gardner, 2015; Rebar et

12 al., 2016). This occurs amid a range of potential personal and contextual confounding or

13 constraining factors (e.g., time, money, availability of facilities, self-efficacy, social

14 support). Unfortunately the increased complexity of interventions can result in poor

15 implementation (Young et al., 2008). This highlights the importance of evaluating

16 implementation in the case of complex interventions; if a behaviour change intervention

17 cannot be effectively delivered in practice then there is little value in pursuing the

18 implementation.

19 Efforts to base interventions on theory may also lead researchers to lose sight of

20 practicality and how such interventions might be adopted and applied in 'real world'

21 situations. Adaptation of interventions to complement the needs and requirements of

22 particular settings has been labelled 'pro-adaptation' and is an approach that has been

23 held for many years (Berman \& Mclaughlin, 1976). This practice is perhaps more

24 relevant to circumstances in which research has an explicit aim to inform policy (Dane

$25 \&$ Schneider, 1998). With the association between academic research and impact 
1 becoming stronger, it is becoming increasingly important to ensure that interventions

2 are sustainable beyond the end of the research project and can be employed by a range

3 of individuals. Evaluating intervention implementation may, therefore, help the research

4 team to identify which components are critical to retain and which are less so, during a

5 process of pro-adaptation.

Many approaches to process evaluation adopt a 'checklist' design in which key

7 components are expected to be evident for the intervention to be considered effective.

8 For example, in the physical activity field, checklists of key components and/or

9 observation sheets to record whether expected behaviours are evident are common tools

10 described in the literature (Fortier, Duda, Guerin, \& Teixeira, 2012; Robbins et al.,

11 2014; Young et al., 2008). These checklists, as well as broader recommendations for

12 checklists such as those of Borelli (2011), may be useful starting points for the design of

13 a fidelity assessment. The popularity of this approach implies an assumption that to be

14 efficacious, an intervention should have standardised and consistent components which

15 can be measured to gauge fidelity, and subsequently and precisely replicated across

16 deliveries by different providers or to different groups. However, we question whether

17 all interventions should be designed to be delivered with $100 \%$ replication of specified

18 criteria. For example, the efficacy of techniques such as motivational interviewing

19 (Miller \& Rollnick, 2002) require the significant other to be able to make ongoing

20 judgements and adaptations to most appropriately respond to individual circumstances.

21 As such, in many interventions perhaps there can be no exact 'formula' that will be

22 effective in promoting autonomous, sustained and healthful engagement in physical

23 activity.

24 An alternative perspective that may be appropriate for interventions based on

25 theories of motivation is to define 'minimal acceptable' guidelines, or critical and non- 
1 critical components (Bauman, Stein, \& Ireys, 1991), and an effectively trained

2 intervention practitioner can decide when it is appropriate or possible to deliver the

3 most relevant components at the appropriate times. For example, an exercise instructor

4 who does not use all possible strategies from a particular theory of motivation in an

5 intervention will not necessarily be ineffective in providing an appropriate 'dose' of the

6 intervention. He or she may determine which intervention components from the theory

7 may be appropriate to include, and which components to exclude, based on experience

8 and, importantly, the available evidence. Formative research is paramount in this regard,

9 and an effectively trained practitioner will know how to use the evidence gained from

10 research to inform the content of their interventions. As an example, consider an

11 intervention based on self-determination theory (Deci \& Ryan, 1985, 2000) aiming to

12 train diabetes nurses to be autonomy supportive when presenting a new physical activity

13 programme to patients. There may seem to be no reason for a nurse to use some features

14 of an autonomy supportive style (e.g., provide a rationale for the activity) if working

15 with an individual patient who has previously expressed his or her autonomous reasons

16 for engaging in the proposed programme and fully endorses their own reasons for

17 completing it. The nurse could still adopt a theoretically appropriate and supportive

18 style and tone, and focus on helping the patient to have further input and decision

19 making into creating the shape of their programme. This could be done in a manner that

20 is optimally challenging and self-referenced, and with a style that emulates care, respect

21 and promotes a sense of belonging. In this case, these would be the 'critical

22 components' relevant to effectiveness of the intervention with this particular patient.

23 The patient could leave with his or her psychological needs fully supported, but many

24 components of autonomy supportive instructing, as specified in the theory, would not

25 have been present in the intervention during this particular interaction. 
The inadequacy of a 'dose-response' approach to understanding motivation has

2 been highlighted in observational work based on self-determination theory (Smith et al.,

3 2015). Consideration of the potency of the motivational climate created by the actions

4 and inactions of a significant other is one recently adopted approach (Smith et al.,

5 2015). From this perspective, what is considered more important is not the number of

6 behaviours exhibited by a significant other, but their psychological meaning, in terms of

7 the anticipated strength of the impact upon the basic needs of the individual. As we

8 have highlighted previously, such considerations make it questionable as to whether it is

9 possible to apply some aspects of 'traditional' models of implementation evaluation that

10 refer to measuring 'dose' and 'treatment' (e.g., Borrelli, 2011). The language derived

11 from medical and/or clinical settings implies that a 'dose - response' relationship is

12 possible, and that 'treatment' can be standardised. Whether or not this is appropriate

13 depends very much on the study design and targeted outcome. For example, drawing

14 from the TPB (Ajzen, 1991) a researcher may consider whether delivery of a health-

15 related message targeting behavioural intentions changes the amount of times an

16 individual chooses to take the stairs over the lift. One could hypothesise that exposure to

17 the message may relate to a quantifiable behavioural outcome (i.e., taking the stairs). As

18 such, 'dose' can be easily quantified by controlling and measuring exposure to the

19 message. However, when the researcher sets out to change the philosophical approach

20 and behaviour of a rheumatoid arthritis nurse by training him/her to apply aspects of

21 TPB to promote realistic intentions to exercise, assessing 'dose' becomes more

22 problematic. Assessments of implementation in such cases need to be designed to

23 operate effectively with the ebb and flow in correspondence with the reality of

24 motivating individuals in social contexts.

\section{Future Research Directions}


One solution in attempting to capture the effectiveness of complex behaviour

2 change interventions is to adopt correspondingly intricate models of process evaluation

3 to capture the complexity and multi-component nature of behaviour change models

4 (Baranowski \& Jago, 2005). However, a thorough process evaluation that attempts to

5 collect data via a range of methods and sources requires significant resource and is,

6 perhaps for this reason, rarely accomplished. It remains the reality that even grant-

7 funded physical activity behaviour change intervention studies rarely have surplus

8 budget beyond what is needed for intervention delivery and measures of effect. As

9 previously identified, some researchers manage this challenge by applying some but not

10 all components of a process evaluation model. In the case of motivation-based physical

11 activity promotion research, it would first be advantageous to establish which

12 components of a theory or model should be prioritised as most relevant and useful in the 13 study of intervention implementation.

14 Second, with an eye on balancing practicality with utility, it would be useful to

15 empirically or otherwise substantiate how much information is enough information to

16 make a clear judgement on quality of intervention implementation and its relevance for

17 study outcomes. For example, coding of practitioners delivering an intervention is one

18 of the more popular methods when evaluating quality of implementation. However, this

19 may be unrealistic in terms of time required as well as intrusion of a researcher or

20 camera being present when an intervention is delivered in naturalistic settings. Future

21 research could serve the field well by examining whether there is a critical percentage

22 or number of sessions that can be observed in order to get 'sufficient' assessment of the

23 quality of implementation, without having to code every event yet still accounting for

24 possible reactivity effects. 
2 ingredients will also impact upon the proposed analysis of intervention effects. If

3 flexibility is to be adopted, it would be challenging to effectively implement per

4 protocol analysis. In such cases, pilot work should be utilised to ascertain which

5 components are critical and could be defined a priori, and which can be considered

6 flexible. One might also argue that intention to treat analysis for physical activity

7 behaviour change trials can offer valuable information regarding the pragmatic value of

8 an intervention when delivered in 'real-life' settings.

Once intervention implementation data have been collected and condensed, the

10 researchers must then decide how to analyse and report these findings. One option is to

11 report findings independent of the main effectiveness paper, within a process evaluation

12 type paper. This is advantageous in that it becomes possible to read a thorough and

13 detailed account of the process evaluation, and to determine whether high fidelity was

14 achieved. However, as proposed in the model put forward by Moore et al. (2015), the

15 different facets of a process evaluation interact with one another, as well as with

16 outcome measures of effectiveness. So if the implementation process is not considered

17 in conjunction with study findings then it is difficult to determine how the quality of

18 intervention consistency in intervention delivery may explain significant or null

19 findings. If type III error is to be avoided, recommendations of how to incorporate

20 process evaluation data into tests of intervention effects would be advantageous.

21 In studies targeting the behaviours or communication style of a significant other,

22 it is important to consider whether their behaviours generate the type of social

23 environment that is motivationally adaptive and supportive of physical activity levels.

24 However, in understanding what has contributed to their effectiveness (or not), it is

25 important too to evaluate the quality and consistency in the training of these individuals 
1 to create the desired motivational environment. Variations in training may explain

2 differences in implementation of the intervention. Often, this is overlooked in the

3 reporting of studies, and could be potentially limiting, in terms of the future translation

4 of findings into practice, or dissemination of effective training strategies between

5 studies in different contexts. We call for researchers to be more explicit in reporting the

6 training process implemented (and make use of online supplementary materials in

7 journals, when such options exist), as well as examining the quality and consistency of

8 implementation of this training. There is also the question of what constitutes 'adequate'

9 training to effectively deliver an intervention. This will require an understanding of the

10 principles of the underlying theory on behalf of the trainee, as well as the ability to

11 utilise these effectively using a range of strategies in expected and spontaneous

12 scenarios. Future research might focus not only on the development of methods that can

13 be used to upskill those whose behaviour we are trying to impact, but also to evaluate

14 the quality of this training and their enactment of the targeted behaviours. Tools such as

15 manuals, implementation guides, reflection, peer networks and mentoring can aid the

16 quality of implementation and their use is commonly reported in the literature. The

17 potential risk of 'drift' in quality of intervention delivery has previously been

18 highlighted (Borrelli, 2011) and this may be partly attributable to disengagement with

19 resources designed to keep the intervention on track. From the perspective of theories of

20 motivation, to be effective and engaging, such methods and resources would need to be

21 designed and implemented in such a way as to be motivationally adaptive. However,

22 this consideration is rarely discussed or reported. Future research could also focus

23 specifically on identifying the most efficacious design and use of intervention support

24 resources and tools that pull from a particular theory which may enhance the 
1 implementer's or end-user's sustained engagement with the resource throughout the

2 intervention.

3 Such data has the potential to expose the weaknesses in the theory, as well as in

4 the intervention itself. Unfortunately, such data are harder to publish and typically, not

5 the outcome desired in reports of grant-funded research. Just as can be the case with

6 study outcome data, intervention implementation data is also at risk of selective

7 publication. In the long term, the quality of interventions will improve if more data were

8 published on what did not work, and not just on what did. This perhaps leads to a call to

9 journal editors to publish more null findings, when they are substantiated with concerted

10 and detailed considerations of why an intervention did not work, as well as informed

11 recommendations for a required change to improve effectiveness.

13 Conclusion

14 We have discussed the challenges faced by researchers who may wish to

15 evaluate intervention implementation in motivation focused physical activity

16 intervention studies. We have also outlined the diversity of approaches that have been

17 adopted in the wider health promotion literature to undertake this task, the paucity of

18 attention this topic has attracted in motivation-focused physical activity intervention

19 studies, as well as the components of implementation that have been utilised in some

20 investigations. We have raised some potential issues with the current diversity of

21 definitions of key terms surrounding intervention implementation and called for

22 movement towards a common interpretation and language. Finally, we have highlighted

23 the potential limitations of translating some of these approaches into work grounded in

24 theories of motivation, without due consideration of the epistemological and conceptual

25 underpinnings of the intended intervention. 
The social-psychological characteristics of many theories of motivation and the

2 diversity of ways these theories are applied may make it impossible to create a 'one size

3 fits all' method or model to evaluate intervention implementation. However, we propose

4 that there are still further steps that could be taken to improve understanding of how

5 theories of motivation can most effectively be applied to maximise the motivational

6 environment in physical activity contexts, and in turn, promote physical activity

7 behaviour change. These include moving towards a common language of

8 implementation, studies to compare the efficacy of several models of implementation,

9 and investigations to help develop guidelines for approaches to measurement of

10 intervention implementation that remain ecologically valid and yet also practical in

11 terms of time, resource and utility in analysis.

12 Theories of motivation have considerable potential to inform physical activity

13 promotion efforts and this is reflected in the diversity of ways the theory is now applied

14 in intervention studies targeting the behaviours of instructors, health professionals,

15 teachers and sport coaches. The on-going development of approaches to evaluate and

16 optimise intervention implementation in a manner that does not lose sight of the essence

17 of the theory (or theories) will be critical to the development of interventions that are

18 effective in promoting physical activity. 
Ajzen, I. (1991). The Theory of Planned Behavior. Organizational Behavior and Human Decision Processes, 50(2), 179-211. doi:Doi 10.1016/07495978(91)90020-T

Albrecht, L., Archibald, M., Arseneau, D., \& Scott, S. D. (2013). Development of a checklist to assess the quality of reporting of knowledge translation interventions using the Workgroup for Intervention Development and Evaluation Research (WIDER) recommendations. Implementation Science, 8, 52.

Bandura, A. (1986). Social foundations of thought and action: A social-cognitive theory. . Englewood Cliffs, NJ: Prentice-Hall.

Baranowski, T., \& Jago, R. (2005). Understanding the mechanisms of change in children's physical activity programs. Exercise and Sport Sciences Reviews, 33(4), 163-168. doi:Doi 10.1097/00003677-200510000-00003

Bardus, M., Blake, H., Lloyd, S., \& Suzanne Suggs, L. (2014). Reasons for participating and not participating in a e-health workplace physical activity intervention: A qualitative analysis. International Journal of Workplace Health Management, 7(4), 229-246.

Basch, C. E., \& Gold, R. S. (1985). The validity of reliability assessments. Journal of School Health, 55(5), 191-195.

Bauman, L. J., Stein, R. E., \& Ireys, H. T. (1991). Reinventing fidelity: the transfer of social technology among settings. American Journal of Community Psychology, 19(4), 619-639.

Bellg, A. J., Borrelli, B., Resnick, B., Hecht, J., Minicucci, D. S., Ory, M., . . . Czajkowski, S. (2004). Enhancing treatment fidelity in health behavior change studies: Best practices and recommendations from the NIH behavior change consortium. Health Psychology, 23(5), 443-451. doi:10.1037/02786133.23.5.443

Berman, P., \& Mclaughlin, M. W. (1976). Implementation of Educational Innovation. Educational Forum, 40(3), 344-370.

Biddle, S., Mutrie, N., \& Gorely, T. (2015). Psychology of physical activity: Determinants, well-being and interventions (Third edition. ed.). Milton Park, Abingdon, Oxon ; New York, NY: Routledge.

Borrelli, B. (2011). The assessment, monitoring, and enhancement of treatment fidelity in public health clinical trials. Journal of Public Health Dentistry, 71, S52-S63. doi:10.1111/j.1752-7325.2011.00233.x

Chatzisarantis, N. L. D., \& Hagger, M. S. (2005). Effects of a brief intervention based on the theory of planned behavior on leisure-time physical activity participation. Journal of Sport \& Exercise Psychology, 27(4), 470-487.

Cohen, K. E., Morgan, P. J., Plotnikoff, R. C., Callister, R., \& Lubans, D. R. (2015). Physical Activity and Skills Intervention: SCORES Cluster Randomized Controlled Trial. Medicine and Science in Sports and Exercise, 47(4), 765-774.

Conner, M. T., \& Norman, P. (2015). Predicting and changing health behaviour: Research and practice with social cognition models (3rd ed.). Maidenhead, UK: Open University Press.

Craig, P., Dieppe, P., Macintyre, S., Michie, S., Nazareth, I., \& Petticrew, M. (2008). Developing and evaluating complex interventions: the new Medical Research Council guidance. British Medical Journal, 337(7676). doi:ARTN a1655

10.1136/bmj.a1655 
Dane, A. V., \& Schneider, B. H. (1998). Program integrity in primary and early secondary prevention: Are implementation effects out of control. Clinical Psychology Review, 18(1), 23-45. doi:Doi 10.1016/S0272-7358(97)00043-3

Deci, E. L., \& Ryan, R. M. (1985). Intrinsic motivation and self-determination in human behavior. New York: Plenum.

Deci, E. L., \& Ryan, R. M. (2000). The "what" and "why" of goal pursuits: Human needs and the self-determination of behavior. Psychological Inquiry, 11(4), 227268. doi:Doi 10.1207/S15327965pli1104_01

Duda, J. L., Quested, E., Haug, E., Samdal, O., Wold, B., Balaguer, I., . . Cruz, J. (2013). Promoting Adolescent health through an intervention aimed at improving the quality of their participation in Physical Activity (PAPA): Background to the project and main trial protocol. International Journal of Sport and Exercise Psychology, 11(4), 319-327. doi:10.1080/1612197X.2013.839413

Dusenbury, L., Brannigan, R., Falco, M., \& Hansen, W. B. (2003). A review of research on fidelity of implementation: implications for drug abuse prevention in school settings. Health Education Research, 18(2), 237-256.

Fortier, M. S., Duda, J. L., Guerin, E., \& Teixeira, P. J. (2012). Promoting physical activity: development and testing of self-determination theory-based interventions. International Journal of Behavioural Nutrition and Physical Activity, 9, 20. doi:10.1186/1479-5868-9-20

Gardner, B. (2015). A review and analysis of the use of 'habit' in understanding, predicting and influencing health-related behaviour. Health Psychology Review, 9(3), 277-295.

Glasgow, R. E., Vogt, T. M., \& Boles, S. M. (1999). Evaluating the public health impact of health promotion interventions: the RE-AIM framework. American Journal of Public Health, 89(9), 1322-1327.

Greaves, C. J., Sheppard, K. E., Abraham, C., Hardeman, W., Roden, M., Evans, P. H., . . . Grp, I. S. (2011). Systematic review of reviews of intervention components associated with increased effectiveness in dietary and physical activity interventions. BMC Public Health, 11. doi:Artn 119

10.1186/1471-2458-11-119

Hagger, M. S., \& Luszczynska, A. (2014). Implementation intention and action planning interventions in health contexts: State of the research and proposals for the way forward. Applied Psychology: Health and Well-Being, 6(1), 1-47.

Hancox, J. E., Quested, E., Thøgersen-Ntoumani, C., \& Ntoumanis, N. (2015). An intervention to train group exercise class instructors to adopt a motivationally adaptive communication style: a quasi-experimental study protocol. Health Psychology and Behavioral Medicine, 3(1), 190-203. doi:10.1080/21642850.2015.1074075

Hardcastle, S. J., Fortier, M. S., Blake, N., \& Hagger, M. S. (In press). Identifying techniques to change motivation and behavior within motivational interviewing and relations with techniques from an existing taxonomy of behavior-change techniques. Health Psychology Review.

Hoddinott, P. (2015). A new era for intervention development studies. Pilot and Feasibility Studies, 1(1), 1-4.

Hoffmann, T. C., Glasziou, P. P., Boutron, I., Milne, R., Perera, R., Moher, D., . . . Johnston, M. (2014). Better reporting of interventions: template for intervention description and replication (TIDieR) checklist and guide. BMJ, 348, g1687.

Kohl, H. W., Craig, C. L., Lambert, E. V., Inoue, S., Alkandari, J. R., Leetongin, G., . . . Workin, L. P. A. S. (2012). The pandemic of physical inactivity: global action 
for public health. Lancet, 380(9838), 294-305. doi:10.1016/S01406736(12)60898-8

Latimer, A. E., Ginis, K. A. M., \& Arbour, K. P. (2006). The efficacy of an implementation intention intervention for promoting physical activity among individuals with spinal cord injury: A randomized controlled trial. Rehabilitation Psychology, 51(4), 273-280. doi:10.1037/0090-5550.51.4.273

Lewis, A. L., \& Eves, F. F. (2012). Testing the theory underlying the success of pointof-choice prompts: A multi-component stair climbing intervention. Psychology of Sport and Exercise, 13(2), 126-132. doi:10.1016/j.psychsport.2011.10.001

Lubans, D. R., Foster, C., \& Biddle, S. J. (2008). A review of mediators of behavior in interventions to promote physical activity among children and adolescents. Preventive Medicine, 47(5), 463-470.

Marcus, B. H., Williams, D. M., Dubbert, P. M., Sallis, J. F., King, A. C., Yancey, A. K., . . Claytor, R. P. (2006). Physical activity intervention studies - What we know and what we need to know - A scientific statement from the American Heart Association Council on Nutrition, Physical Activity, and Metabolism (Subcommittee on Physical Activity); Council on Cardiovascular Disease in the Young; and the Interdisciplinary Working Group on Quality of Care and Outcomes Research. Circulation, 114(24), 2739-2752. doi:10.1161/Circulationaha.106.179683

Michie, S. (2008). What works and how? Designing more effective interventions needs answers to both questions. Addiction, 103(6), 886-887.

Michie, S., \& Abraham, C. (2004). Identifying techniques that promote health behaviour change: Evidence based or evidence inspired. Psychology and Health, 19, 29-49.

Michie, S., Hardeman, W., Fanshawe, T., Prevost, A. T., Taylor, L., \& Kinmonth, A. L. (2008). Investigating theoretical explanations for behaviour change: The case study of ProActive. Psychology and Health, 23(1), 25-39.

Michie, S., van Stralen, M. M., \& West, R. (2011). The behaviour change wheel: a new method for characterising and designing behaviour change interventions. Implement Sci, 6, 42. doi:10.1186/1748-5908-6-42

Miller, W. R., \& Rollnick, S. (2002). Motivational interviewing: Preparing people for change (2nd ed.). New York: Guilford Press.

Moore, G. F., Audrey, S., Barker, M., Bond, L., Bonell, C., Hardeman, W., . . Baird, J. (2014). Process evaluation of complex interventions: UK Medical Research Council (MRC) guidance. Retrieved from

Moore, G. F., Audrey, S., Barker, M., Bond, L., Bonell, C., Hardeman, W., ... Baird, J. (2015). Process evaluation of complex interventions: Medical Research Council guidance. Bmj-British Medical Journal, 350. doi:ARTN h1258

10.1136/bmj.h1258

Murray, A., Hall, A. M., Williams, G. C., McDonough, S. M., Ntoumanis, N., Taylor, I. M., . . L Lonsdale, C. (2015). Effect of a Self-Determination Theory-Based Communication Skills Training Program on Physiotherapists' Psychological Support for Their Patients With Chronic Low Back Pain: A Randomized Controlled Trial. Archives of Physical Medicine and Rehabilitation, 96(5), 809816. doi:10.1016/j.apmr.2014.11.007

Naylor, P.-J., Nettlefold, L., Race, D., Hoy, C., Ashe, M. C., Higgins, J. W., \& McKay, H. A. (2015). Implementation of school based physical activity interventions: a systematic review. Preventive Medicine, 72, 95-115. 
Ntoumanis, N., Thøgersen-Ntoumani, C., Quested, E., \& Hancox, J. (2016). The effects of training group exercise class instructors to adopt a motivationally adaptive communication style. Scandinavian Journal of Medicine \& Science in Sports.

Prestwich, A., Sniehotta, F. F., Whittington, C., Dombrowski, S. U., Rogers, L., \& Michie, S. (2014). Does theory influence the effectiveness of health behavior interventions? Meta-analysis. Health Psychology, 33(5), 465-474. doi: $10.1037 / \mathrm{a} 0032853$

Rebar, A. L., Dimmock, J. A., Jackson, B., Rhodes, R. E., Kates, A., Starling, J., \& Vandelanotte, C. (2016). A systematic review of the effects of non-conscious regulatory processes in physical activity. Health Psychology Review, 1-86.

Rhodes, R. E., \& Dickau, L. (2012). Experimental evidence for the intention-behavior relationship in the physical activity domain: A meta-analysis. Health Psychology, 31(6), 724.

Rhodes, R. E., \& Pfaeffli, L. A. (2010). Review Mediators of physical activity behaviour change among adult non-clinical populations: a review update. International Journal of Behavioural Nutrition and Physical Activity, 7(37).

Robbins, L. B., Pfeiffer, K. A., Wesolek, S. M., \& Lo, Y. J. (2014). Process evaluation for a school-based physical activity intervention for 6th-and 7th-grade boys: Reach, dose, and fidelity. Evaluation and Program Planning, 42, 21-31. doi:10.1016/j.evalprogplan.2013.09.002

Smith, J. J., Morgan, P. J., Plotnikoff, R. C., Dally, K. A., Salmon, J., Okely, A. D., . . Lubans, D. R. (2014). Smart-phone obesity prevention trial for adolescent boys in low-income communities: the ATLAS RCT. Pediatrics, 134(3), e723-e731.

Smith, N., Tessier, D., Tzioumakis, Y., Quested, E., Appleton, P., Sarrazin, P., . . . Duda, J. L. (2015). Development and Validation of the Multidimensional Motivational Climate Observation System. Journal of Sport \& Exercise Psychology, 37(1), 4-22. doi:10.1123/jsep.2014-0059

Young, D. R., Steckler, A., Cohen, S., Pratt, C., Felton, G., Moe, S. G., . . Raburn, B. (2008). Process evaluation results from a school- and community-linked intervention: the Trial of Activity for Adolescent Girls (TAAG). Health Education Research, 23(6), 976-986. doi:10.1093/her/cyn029 